\title{
Preface to the special issue dedicated to the 6th World Congress on Global Optimization held in Metz, France, July 8-10, 2019
}

\author{
Hoai An Le Thi ${ }^{1} \cdot$ Tao Pham Dinh ${ }^{2} \cdot$ Yaroslav D. Sergeyev ${ }^{3,4}$
}

Received: 5 January 2021 / Accepted: 12 February 2021 / Published online: 25 February 2021

(c) The Author(s), under exclusive licence to Springer-Verlag GmbH, DE part of Springer Nature 2021

The World Congress on Global Optimization (WCGO) is the premier biennial event of the International Society of Global Optimization (ISoGO). The 6th WCGO was organized in Metz, France on July 8-10, 2019, by the Computer Science and Applications Department (IA), LGIPM, University of Lorraine. The previous congresses were organized in Changsha, Hunan, China (2009), Chania, Greece (2011), Huangshang, China (2013), Gainesville, Florida, USA (2015), and College Station, TX, USA (2017).

WCGO 2019 aimed to bring together most leading specialists in both theoretical and algorithmic aspects as well as a variety of application domains of global and nonconvex optimization to highlight recent advances, trends, challenges and discuss how to expand the role of these fields in several potential high-impact application areas.

WCGO 2019 was attended by about 180 scientists and practitioners from 40 countries. The scientific program included the oral presentation of 112 selected full papers as well as several selected abstracts covering all main research areas in global optimization. In addition, the conference program was enriched by six plenary lectures that were given by Prof. Aharon Ben-Tal (Israel Institute of Technology, Israel), Prof. Immanuel M. Bomze (University of Vienna, Austria), Prof. Masao Fukushima (Nanzan University, Japan), Prof. Anna Nagurney (University of Massachusetts Amherst, USA), Prof. Panos M. Pardalos (University of Florida, USA), and Prof. Anatoly Zhigljavsky (Cardiff University, UK). One of the highlights of the WCGO 2019 was the announcement of the winners of the Constantin Carathéodory Prize of the ISoGO. The Prize was awarded to Profs. Anna Nagurney and Prof.

Yaroslav D. Sergeyev

yaro@dimes.unical.it

1 Universite de Lorraine, Metz, France

2 Institut National Des Sciences Appliquees de Rouen, Rouen, France

3 University of Calabria, Rende, CS, Italy

4 Lobachevsky State University, Nizhni Novgorod, Russia 
Anatoly Zhigljavsky in recognition of their lifetime contributions to the field of global optimization.

The conference proceedings book was published in the series Advances in Intelligent Systems and Computing of Springer-Verlag and indexed by ISI Proceedings, DBLP, Ulrich's, EI-Compendex, SCOPUS, Zentralblatt Math, MetaPress, and Springerlink. The book contains 112 papers selected from about 250 submissions to WCGO 2019. Each paper was peer-reviewed by at least two members of the International Program Committee and the International Reviewer Board. The book covers both theoretical and algorithmic aspects of global optimization and nonconvex programming, as well as its applications to modeling and solving decision problems in various domains.

A collection of the extended versions of contributions was selected to be published in the three special issues in Journal of Global Optimization, Optimization Letters, and Optimization in Engineering. This special issue contains eight papers that have been rigorously peer-reviewed by at least two reviewers and are briefly described below. The main group of papers of the special issue deals with a number of aspects and problems arising in the framework of DC programming. There are the following three papers dealing with this subject: (i) A. Ghazi, A. Roubi "Optimality conditions and DC-Dinkelbach-type algorithm for generalized fractional programs with ratios of difference of convex functions"; (ii) Thi Thuy Tran, Hoai An Le Thi, Tao Pham Dinh, Nhu Tuan Nguyen "DC programming and DCA for enhancing physical layer security via relay beamforming strategies"; (iii) Yi-Shuai $\mathrm{Niu}, \mathrm{Yu}$ You, Wenxu Xu, Wentao Ding, Junpeng Hu, Songquan Yao “A differenceof-convex programming approach with parallel branch-and-bound for sentence compression via a hybrid extractive model".

The article "Two-machine flow shop with dynamic storage space" written by J. Berlinska, A. Kononov, and Y. Zinder considers shop scheduling problems with job dependent storage requirements where the availability of the storage space is a function of time. It strengthens the previously published result concerning the existence of an optimal permutation schedule and presents a polynomial-time approximation scheme together with several heuristic algorithms. The paper M. Cococcioni, L. Fiaschi "The Big-M method with the numerical infinite M" uses the recently proposed Grossone methodology to introduce a non-parametric variant of the Big-M method able to overcome the drawbacks of its classical counterpart (mainly, the difficulty in setting the right value for the constant $\mathrm{M}$ ). The paper "Computationally efficient approach for solving lexicographic multicriteria optimization problems" written by V. Gergel, E. Kozinov, and K. Barkalov proposes an approach to work with lexicographic problems where criteria can be multiextremal and hard to be evaluated.

In the article "Existence results for a dynamic Sturm-Liouville boundary value problem on time scales" the authors S. Heidarkhani, S. Moradi, and G. Caristi apply variational methods to prove the existence of infinitely many local minima for the Euler functional corresponding to the considered boundary value problem. Finally, the paper M. Hladık, D. Hartman, M. Zamani "Maximization of a PSD quadratic form and factorization" focuses on finding upper bounds on the maximum value investigating usefulness of different vector norms and different objective matrix 
factorizations arriving at some kind of duality with positive duality gap but with possibly tight bounds.

We conclude this Preface by highlighting that the conference was organized by the Computer Science and Applications Department, LGIPM, University of Lorraine, France. We wish to especially thank all members of the Scientific and Organizing Committees for their excellent work to make the conference a success. The conference would not have been possible without their considerable effort. We would like to express our sincere thanks to our main sponsors: Réseau de Transport d'Électricité (France), Conseil régional du Grand Est (France), Metz Métropole (France), Conseil départemental de la Moselle (France), Université de Lorraine (France), Laboratoire de Génie Informatique, de Production et de Maintenance (LGIPM)-Université de Lorraine, UFR Mathématique Informatique Mécanique Automatique-Université de Lorraine, VINGROUP and DCA Solutions (Vietnam). 